\title{
Utility of Solid-State UV/Vis Spectra on the Evaluation of Photostability of Indomethacin Crystals
}

\author{
Akihito Kiguchiya, ${ }^{*, a}$ Reiko Teraoka, ${ }^{b}$ and Toshiyasu Sakane ${ }^{b}$ \\ ${ }^{a}$ Technology Research \& Development Division, Sumitomo Dainippon Pharma Co., Ltd.; 3-1-98 Kasugade-naka, \\ Konohana-ku, Osaka 554-0022, Japan: and ${ }^{b}$ Laboratory of Pharmaceutical Technology, Kobe Pharmaceutical \\ University; 4-19-1 Motoyamakita-machi, Higashinada-ku, Kobe 658-8558, Japan. \\ Received May 22, 2018; accepted June 11, 2018
}

Evaluating the stability of drugs over ranges of various environmental conditions is necessary to ensure the quality of the drug throughout the shelf life. In this study, we used indomethacin, which is well known as a photosensitive drug, and evaluated the photostability of prepared crystals. HPLC analysis revealed that all the indomethacin crystals were degraded by light exposure. However, the indomethacin crystals involving solvates had different degrees of photodegradation and different solid-state $U V / V i s$ spectra. The value of the absorption integral in the UVA range related closely to the photodegradation of indomethacin crystals involving solvates. Therefore, it is easy to compare the photosensitivity among the crystals without actual analytical data, by use of a suitable analytical method and using light exposure samples. Moreover, it is possible to predict the value of photodegradation ratio from the solid-state UV/Vis spectra of indomethacin crystals. Therefore, this method may provide key information for selecting the most appropriate crystal form of photosensitive drugs.

Key words photostability; solid-state UV/Vis spectra; stability prediction; UVA region; indomethacin

The purpose of stability testing for pharmaceutical products is to obtain data on how their quality changes with time under a variety of environmental conditions such as temperature, humidity and light exposure, and to establish a shelf life for a drug under the most appropriate storage conditions. ${ }^{1)}$ Storage of samples under various conditions and appropriate chromatographic methods are required to evaluate the stability for each drug properly as there are many differences in the physicochemical properties and chemical structure. ${ }^{2-4)}$ Since it takes at least a few months to prepare the degradation samples and to develop analytical methods for them, it is difficult to shorten the period to evaluate their properties. Therefore, stability studies may sometimes delay the start of clinical studies.

The number of photosensitive drugs has been increasing recently, and many photodegradation mechanisms have been reported. $^{5-8)}$ Additionally, differences in physicochemical properties among crystals have the influences on in-vivo absorption, stability, formulation, manufacturing process, and photosensitivity. ${ }^{9-12)}$ However, it is difficult to compare the photosensitivity of crystals without obtaining their degradation data using appropriate analytical methods. Therefore, a novel method of predicting photostability of a compound is required to enable an easy comparison of photostability.

In this study, we focused on the transition from ground state to excited state of compounds following light absorption. In the Japanese Pharmacopoeia Seventeenth Edition, indomethacin (IMC) is described as being colored by light exposure. Therefore, we evaluated the relationship between photostability and the integral of UV/Vis solid-state spectra in IMC crystals.

\section{Results and Discussions}

IMC Crystals The powder X-ray diffraction (PXRD) pattern of each IMC crystal are shown in Fig. 1. Each PXRD pattern was different from each other. IMC (I) which was reported as IMC $(\gamma)$ was commercial form and IMC (II) was confirmed as IMC $(\alpha)$, based on the results of PXRD pattern and thermal analysis. ${ }^{13)}$ This is the first time to report IMC (III) and IMC (IV). Regarding these two IMC crystals, the weight loss, meaning elimination of each solvent used in preparation, was observed in thermal analysis data (Fig. 2). Thus, it was suggested that IMC (III) and IMC (IV) were solvates with each solvent used in the preparation. From the results of thermal analysis, each stoichiometry of IMC and each solvent of IMC (III) and IMC (IV) is approximately $3: 1$, respectively.

Tablets of IMC crystals were used to collect the solid-state $\mathrm{UV} / \mathrm{Vis}$ spectra to avoid the influence of the particle size on the basis of previous results. ${ }^{14)}$ As shown in Fig. 3, each IMC crystal had a different solid-state UV/Vis spectrum. IMC (I) had a shorter maximum absorption wavelength and small absorbance. On the other hand, the maximum absorption wavelength of IMC solvates (III and IV) were shifted to longer wavelength and the absorbance increased.

Photostability The smaller the particle size of the compound, the larger the impact of the light on the compound, generally. Therefore, IMC tablets were used to evaluate the photostability to avoid the influence of the particle size. As a result of light exposure, the color of all IMC tablets changed from white or off-white to yellow. However, no transformation of crystal form was observed in all IMC crystals based on PXRD patterns. The exposed samples and the control samples of all IMC crystals were analyzed by HPLC to evaluate the degree of photodegradation. The results are shown in Table 1. Comparing the assay values of exposed samples and control samples, the assay values for all IMC crystals decreased due to the influence of light exposure. Moreover, the degree of photodegradation differed among IMC crystals.

Relation between Photosensitivity and UV/Vis Spectra Photodegradation of compounds is caused by light absorption. Thus, it was suggested that the larger the absorption value 


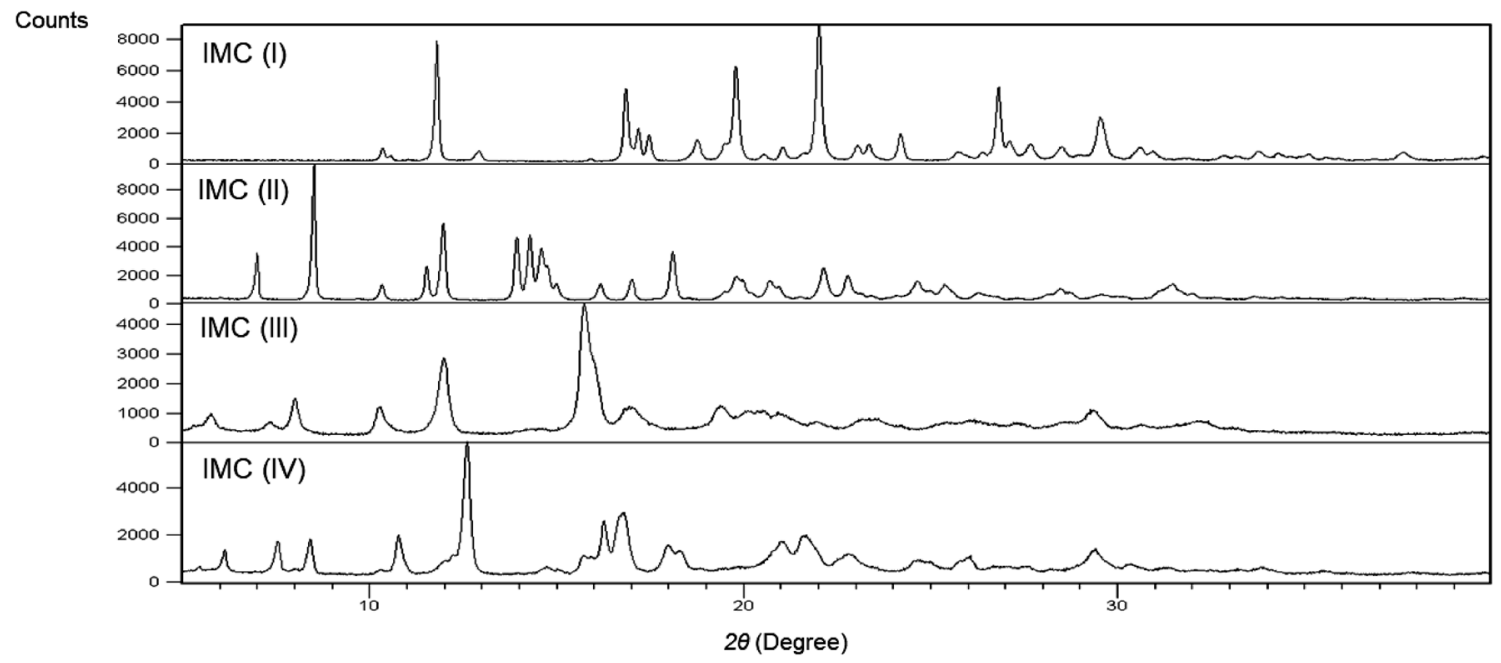

Fig. 1. PXRD Patterns of IMC Crystals

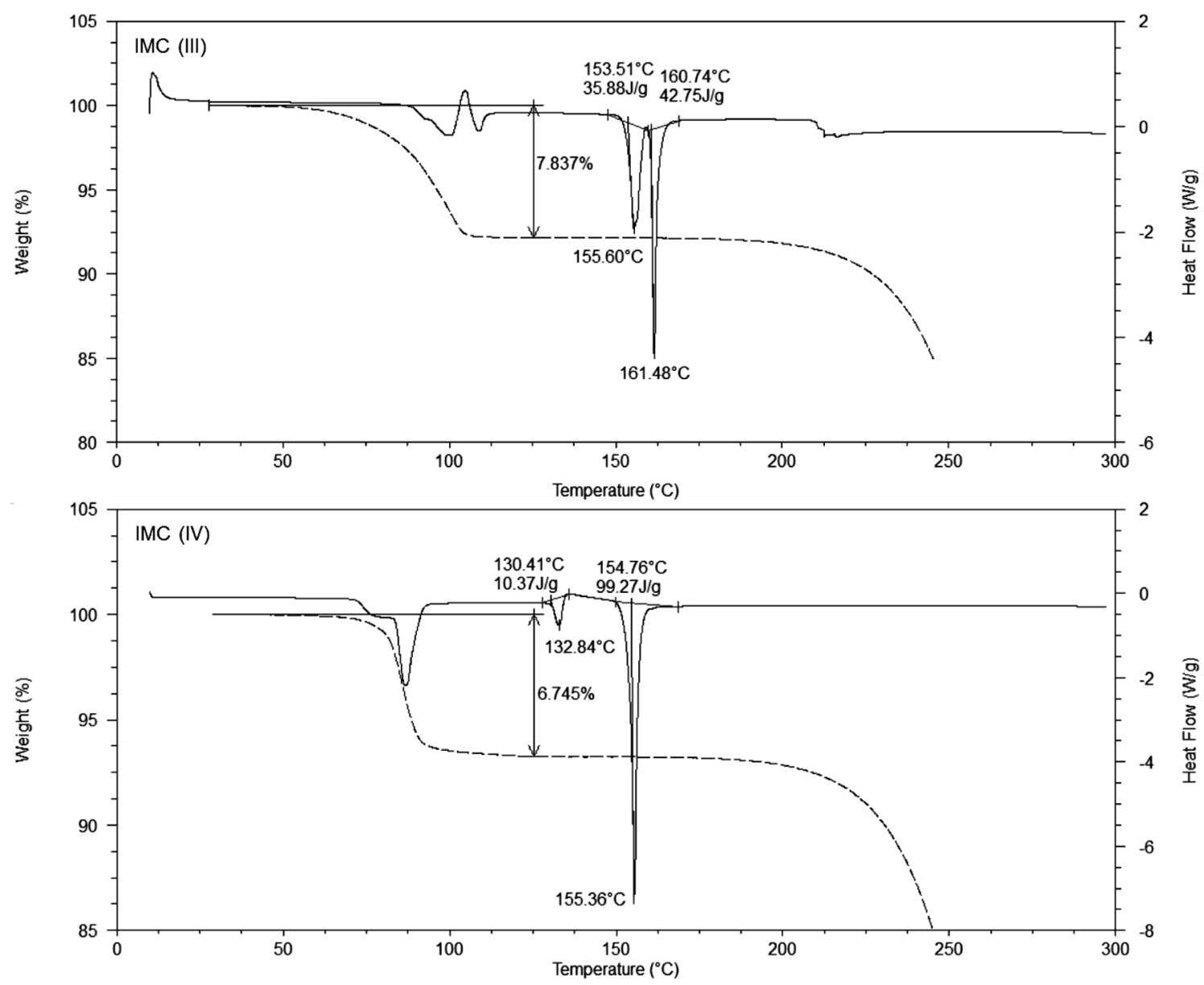

Fig. 2. Thermal Analysis Data for IMC (III) and IMC (IV)

The solid line and dash line represent heat flow (w/g) and weight (\%), respectively.

of compounds, the more photosensitive they were. IMC had characteristic absorption between 300 and $500 \mathrm{~nm}$. The integral values of solid-state UV/Vis spectra over two ranges are shown in Table 1. The IMC crystals with higher photosensitivity also tended to have larger integral values for two ranges. As for the IMC crystals, it is suggested that the integrated $\mathrm{UV} / \mathrm{Vis}$ absorption values closely relate to the degree of pho- todegradation. Plotting the logarithm of the degradation ratio on the ordinate axis against the integral values of solid-state $\mathrm{UV} / \mathrm{Vis}$ spectra over two ranges on the abscissa axis, good relationship between the two was observed as shown in Fig. 4.

D65 lamp, which was the light source in the photoirradiation test, emits light over $300 \mathrm{~nm}$. The importance of the UVA range from 320 to $400 \mathrm{~nm}$ when evaluating photostabil- 


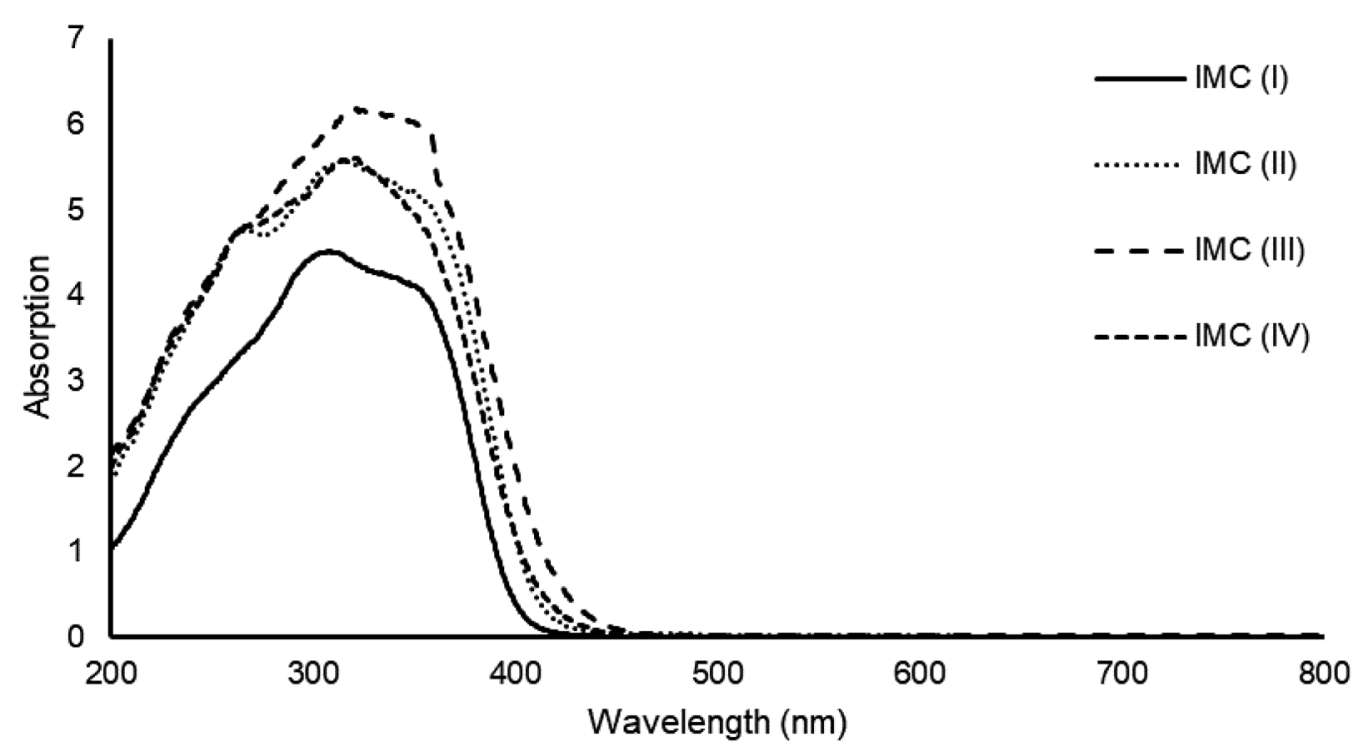

Fig. 3. Solid-state UV/Vis Spectra of IMC Crystals

Table 1. Assay Value after Exposure or No-exposure Sample and Integral Value of UV/Vis Spectra

\begin{tabular}{|c|c|c|c|c|c|}
\hline \multirow{2}{*}{ Sample } & \multicolumn{2}{|c|}{ Assay value $(\%)$} & \multirow{2}{*}{$\begin{array}{l}\text { Photodegradation ratio } \\
\text { (\%) }\end{array}$} & \multicolumn{2}{|c|}{ Integral value of solid-state UV/Vis spectra } \\
\hline & No exposure & Exposure & & $300-500 \mathrm{~nm}$ & $320-400 \mathrm{~nm}$ \\
\hline IMC (I) & 99.65 & 98.75 & 0.90 & 727.8 & 532.8 \\
\hline IMC (II) & 97.18 & 95.41 & 1.77 & 941.9 & 694.1 \\
\hline IMC (III) & 99.80 & 94.69 & 5.11 & 1088.4 & 784.7 \\
\hline IMC (IV) & 99.69 & 97.17 & 2.52 & 877.8 & 634.7 \\
\hline
\end{tabular}

ity is also described in the ICH Q1B guideline, which is the internationally harmonized guideline for the evaluation of photostability of drugs. ${ }^{4)}$ Thus, it was suggested that the UVA range was suitable range to evaluate the photostability of IMC crystals.

Only one report regarding the evaluation of the photostability with solid-state UV/Vis spectra has been published. ${ }^{10)}$ That report describes the first adaptation of polymorphs of tamoxifen citrate to photostability evaluation, and evaluation involving shifting of the UV/Vis absorption. On the other hand, the method of the present study is applicable not only to photostability evaluation of anhydrous forms, but also to solvates, using integral value involving UVA range. It could be possible to predict the photostability of other compounds by comparing the integral values of solid-state UV/Vis spectra, without need for the results of HPLC analysis.

\section{Conclusion}

The utility of this method using solid-state UV/Vis spectra to predict the photosensitivity of IMC crystals was shown. The reliability of this method was corroborated by the results from HPLC data. In the case of IMC crystals, this method with a suitable regression line enables us to estimate the photodegradation rate among the crystals without additional HPLC data. This evaluation method can be adapted to IMC crystals, including anhydrates and solvates. Therefore, this method may provide key information for selecting the most appropriate crystal form of a photosensitive drug substance.

\section{Experimental}

Materials IMC was purchased from FUJIFILM Wako Pure Chemical Corporation (Osaka, Japan). Methanol and toluene were purchased from Nacalai Tesque (Kyoto, Japan). The other solvents, reagents and barium sulfate were purchased from FUJIFILM Wako Pure Chemical Corporation. Water was obtained from a Milli-Q gradient system (Merck, NJ, U.S.A.).

Preparation of Crystals IMC (II) was obtained by heating an ethanol solution of IMC (I) (commercial form) at $80^{\circ} \mathrm{C}$ and adding water stored at room temperature in accordance with a previous report. ${ }^{13)}$ The solid was then obtained by filtration and dried under vacuum at room temperature. IMC (III) and IMC (IV) were obtained by heating a toluene solution and a tetrahydrofuran (THF) solution of IMC (I) at $80^{\circ} \mathrm{C}$ and cooling slowly to room temperature, respectively. The solids obtained were then filtered and dried under vacuum at room temperature.

PXRD Measurement PXRD patterns were obtained with a D8 ADVANCE X-ray diffractometer (Bruker, MA, U.S.A.) at room temperature. X-rays were generated with $\mathrm{CuK \alpha}$ radiation at $40 \mathrm{kV}$ and $40 \mathrm{~mA}$. IMC tablets of each IMC crystal were pressed by pelletizer and placed on reflection-free sample plates. The data were collected from $5^{\circ}$ to $40^{\circ}(2 \theta)$ with a step size of $0.015^{\circ}$ and time per step of $0.25 \mathrm{~s}$.

Thermal Analysis Differential scanning calorimetry (DSC) data were obtained using a DSC Q1000 differential scanning calorimeter (TA Instrument, DE, U.S.A.). Each sample was analyzed in a crimped aluminum pan with a 

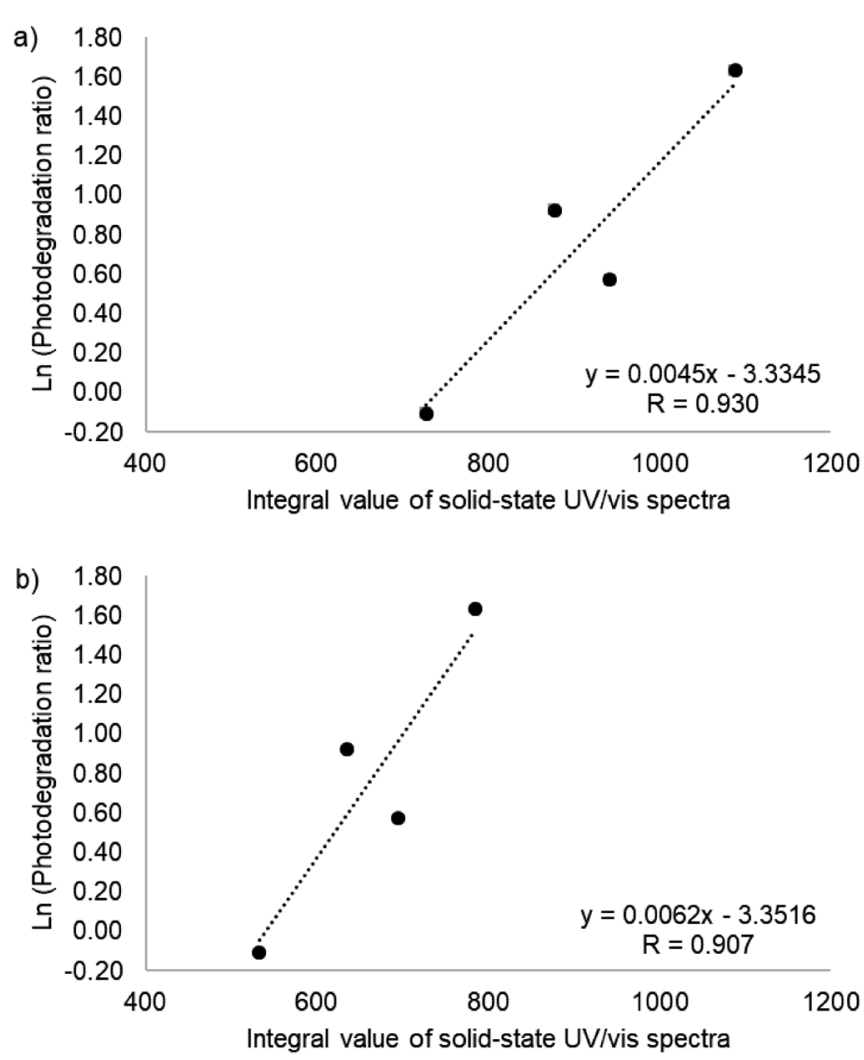

Fig. 4. Relationship between Photodegradation Ratio and Integral Value of Solid-State UV/Vis Spectra

a) $300-500 \mathrm{~nm}$, b) $320-400 \mathrm{~nm}$ (UVA range).

pinhole, heated under a nitrogen purge at a rate of $10^{\circ} \mathrm{C} / \mathrm{min}$, from 10 to $300^{\circ} \mathrm{C}$. Thermogravimetric analysis (TGA) data were obtained with a TGA Q500 thermogravimetric analyzer (TA Instruments). Each sample was analyzed on a platinum pan, heated under a nitrogen purge at a rate of $10^{\circ} \mathrm{C} / \mathrm{min}$, from room temperature to $300^{\circ} \mathrm{C}$.

Photoirradiation Test A light irradiation tester, LT-120A-WCD (Nagano Science, Osaka, Japan), was used to prepare light exposure samples. D65 lamp (Nagano Science) was used as the light source. Two tablets of each IMC crystal were put in separate dishes, and one was wrapped in aluminum foil to provide a dark control. Samples were exposed to light providing an overall illumination of 1.2 million lux hours and an integrated near-UV energy of not less than $200 \mathrm{~W} \mathrm{~h} /$ square meter at room temperature, in accordance with the $\mathrm{ICH}$ Q1B guideline. ${ }^{4)}$
UV/Vis Spectrometry Solid-state UV/Vis spectra were obtained with a UV-2450 system (Shimadzu, Kyoto, Japan) equipped with an integrating sphere unit (ISR-240, Shimadzu) at room temperature. The tablets of each IMC crystal were placed in sample cells. Barium sulfate was used as the reference standard. The spectra were acquired with a $0.5-\mathrm{nm}$ sampling pitch in the wavelength range from 200 to $800 \mathrm{~nm}$. The obtained reflection data were converted to absorption data using the Kubelka-Munk function.

HPLC Analysis The chromatograms were obtained with an HPLC, LC-20A series (Shimadzu) with a reverse phase column, SunFire C18 (Waters, MA, U.S.A.). The UV detector was set at $317 \mathrm{~nm}$, which was the wavelength of maximum absorption for IMC. Mixtures of water including $0.05 \%$ trifluoroacetic acid, and acetonitrile including $0.05 \%$ trifluoroacetic acid were used as the mobile phases. Column temperature was $30^{\circ} \mathrm{C}$ and flow rate was $1 \mathrm{~mL} / \mathrm{min}$. After light irradiation, exposure samples and authentic samples were dissolved in acetonitrile/water and injected into the HPLC system to evaluate the degree of degradation caused by light exposure.

Conflict of Interest The authors declare no conflict of interest.

\section{References}

1) ICH Harmonised Tripartite Guideline Q1A (R2), "Stability Testing of New Drug Substances and Products," February, 2003.

2) ICH Harmonised Tripartite Guideline Q1E, "Evaluation for Stability Data," February, 2003.

3) Waterman K. C., AAPS PharmSciTech, 12, 932-937 (2011).

4) ICH Harmonised Tripartite Guideline Q1B, "Stability Testing: Photostability Testing of New Drug Substances and Products," November, 1996.

5) Akimoto K., Inoue K., Sugimoto I., Chem. Pharm. Bull., 33, 40504053 (1985).

6) Glass B. D., Nov'ak C., Brown M. E., J. Therm. Anal. Calorim., 77, 1013-1036 (2004).

7) Nord K., Andersen H., Tønnesen H. H., Drug Stab., 1, 243-248 (1997).

8) Teraoka R., Otsuka M., Matsuda Y., Int. J. Pharm., 286, 1-8 (2004).

9) Gwak H. S., Choi J. S., Choi H. K., Int. J. Pharm., 297, 156-161 (2005).

10) Kojima T., Onoue S., Katoh F., Teraoka R., Matsuda Y., Kitagawa S., Tsuhako M., Int. J. Pharm., 336, 346-351 (2007).

11) Otsuka M., Hasegawa H., Matsuda Y., Chem. Pharm. Bull., 45, 894-898 (1997).

12) Kitamura M., CrystEngComm, 11, 949-964 (2009).

13) Kaneniwa N., Otsuka M., Hayashi T., Chem. Pharm. Bull., 33, 3447-3455 (1985).

14) Teraoka R., Otsuka M., Matsuda Y., Int. J. Pharm., 184, 35-43 (1999). 